Jede Sekunde zählt. Schon dieser Satz zeigt klar auf, dass im Jahre 2015 der Beginn einer Reanimation durch Laien ein unverzichtbares Element im Algorithmus einer erfolgreichen Reanimation im öffentlichen Raum geworden ist. Nach dem Schock folgt die Aktion, aber leider bleibt es sehr oft beim Schock - und eine rettende Aktion bleibt sehr häufig aus. Wie kann man also bei Laien die Ängste abbauen? Das geht nur, wenn man erstens schon im jungen Alter beginnt, darüber zu sprechen, und zweitens durch Üben, Üben, Üben. Immer mehr an Bedeutung gewinnen technische Hilfsmittel wie Defibrillatoren, wobei man sich gerade hier aufgrund von Unkenntnis und fehlender Schulung gerne in falscher Sicherheit wiegt. Bei einem gezielten Einsatz Leben rettend, kann ein Defibrillator auch behindernd sein, wenn man sich einzig auf dieses Element fokussiert. Koordination und Schulung sind die Herausforderungen von heute und morgen.

Dr. med. Remo Osterwalder, Mitglied des Zentralvorstandes der FMH, Departementsverantwortlicher Rettungswesen

\title{
Reanimation durch Ersthelfer
}

\author{
Patrick Siebenpfund ${ }^{\mathrm{a}}$, Gabriela Kaufmann ${ }^{\mathrm{b}}$, Roman Burkart ${ }^{\mathrm{c}}$, Wolfgang Ummenhofer ${ }^{\mathrm{d}}$ \\ ${ }^{a}$ Dr. med., Facharzt Anästhesiologie FMH, Intensivmedizin FMH, Notarzt SGNOR, Kantonsspital Baselland, Standort Bruderholz \\ ${ }^{b}$ Geschäftsführerin SGNOR und SRC \\ c Dipl. Pflegeexperte, wissenschaftlicher Mitarbeiter Fondazione Ticino Cuore \\ ${ }^{d}$ Prof. Dr. med., Departement Anästhesie, Universitätsspital Basel
}

Die Plattform Rettungswesen der FMH ist das Forum aller ärztlicher Fachgesellschaften, die an der Notfallversorgung vom Ereignisort bis ins Spital (= Rettungskette) beteiligt sind. Im «Leitbild der FMH zum Rettungswesen in der Schweiz» hat sich die Plattform Rettungswesen im Jahr 2010 für eine Verbesserung der Rettungskette ausgesprochen. Im vorliegenden Artikel wird schwerpunktmässig auf die Thematik Reanimation und Basic Life Support (BLS) durch Ersthelfer eingegangen, eine Grundausbildung sollte jedoch sinnvollerweise immer auch in Erster Hilfe stattfinden. Bei einem lebensbedrohlichen Notfall verstreicht vom Zeitpunkt des Ereignisses bis zur Alarmierung und in der Folge auch bis zum Eintreffen der Rettungskräfte viel Zeit. Für den Kreislaufstillstand ist gut belegt, dass der Zeitpunkt des Reanimationsbeginns und die Qualität der Reanimation den Outcome des Patienten [1] nachhaltig bestimmen; dies gilt sowohl für das Überleben an sich als auch für die mögliche neurologische Beeinträchtigung.

\section{Outcome durch flächendeckende Reanimationsausbildung verbessern}

Der Interverband für Rettungswesen (IVR) hat den Zeitraum ab Alarmierung definiert, in welchem ein Patient erreicht werden muss. Die Hilfsfrist ist auf dem Land 15 Minuten, in der Stadt 10 Minuten - jeweils in 90\% aller Fälle. Im Falle eines Kreislaufstillstands ist offensichtlich, dass ohne eine Reanimation durch Ersthelfer - trotz dieser guten Hilfsfristen - häufig jede professionelle Hilfe zu spät kommt. Die sog. Utsteindaten [2] belegen, dass nur etwa $6-7 \%$ der Patienten überleben, die einen Kreislaufstillstand erlitten haben (neurologischer Outcome Grad 1-2) [3]. Diese Zahl hat sich trotz grosser Ausbildungsanstrengungen leider in den letzten drei Dekaden weltweit nicht verbessert, von wenigen Ausnahmen in speziellen Regionen abgesehen. Der schlechte Outcome kann durch eine Ersthelfer-Reanimation massiv verbessert werden. Der Kanton Tessin stellt in dieser Hinsicht den Schweizer Vorzeigekanton dar: Durch vielfältige Unterstützungsprogramme in der Laienrettung sind hier die Outcomezahlen bereits auf ein erstaunliches Überleben von über $40 \%$ angestiegen. Erklärtes Ziel ist es, die Erfolgsrate sogar auf $50 \%$ zu verbessern.

Nur wenn ein plötzlicher Kreislaufstillstand sofort erkannt wird, bestehen für den Patienten realistische Überlebenschancen.

In der Schweiz hat der Swiss Resuscitation Council (SRC) Reanimationsempfehlungen für die Öffentlichkeit ausgearbeitet; leider ist dieses Thema zu wenig prominent, um mit hoher Priorität von den politischen Verantwortlichen an die Hand genommen zu werden. Im Zentrum aller Bemühungen muss die flächendeckende Ausbildung der Bevölkerung in den Basismassnahmen der Reanimation stehen. Das bedeutet, dass in einem Notfall idealerweise immer eine in BLS geschulte Person aus dem nahen Umfeld sofort Hilfe leisten kann, lange bevor die Rettungsdienste vor Ort eintreffen. Die Einführung von BLS-Kursen an Schulen und in Betrieben, der Aufbau von sogenannten First-Responder-Systemen [4] und die unterstützende BLS-Instruktion am Telefon durch den jeweiligen Mitarbeiter der zuständigen Sanitätsnotrufzentrale (SNZ) sind erste Schritte in die richtige Richtung - allerdings nur auf begrenzter kantonaler oder kommunaler Ebene. 


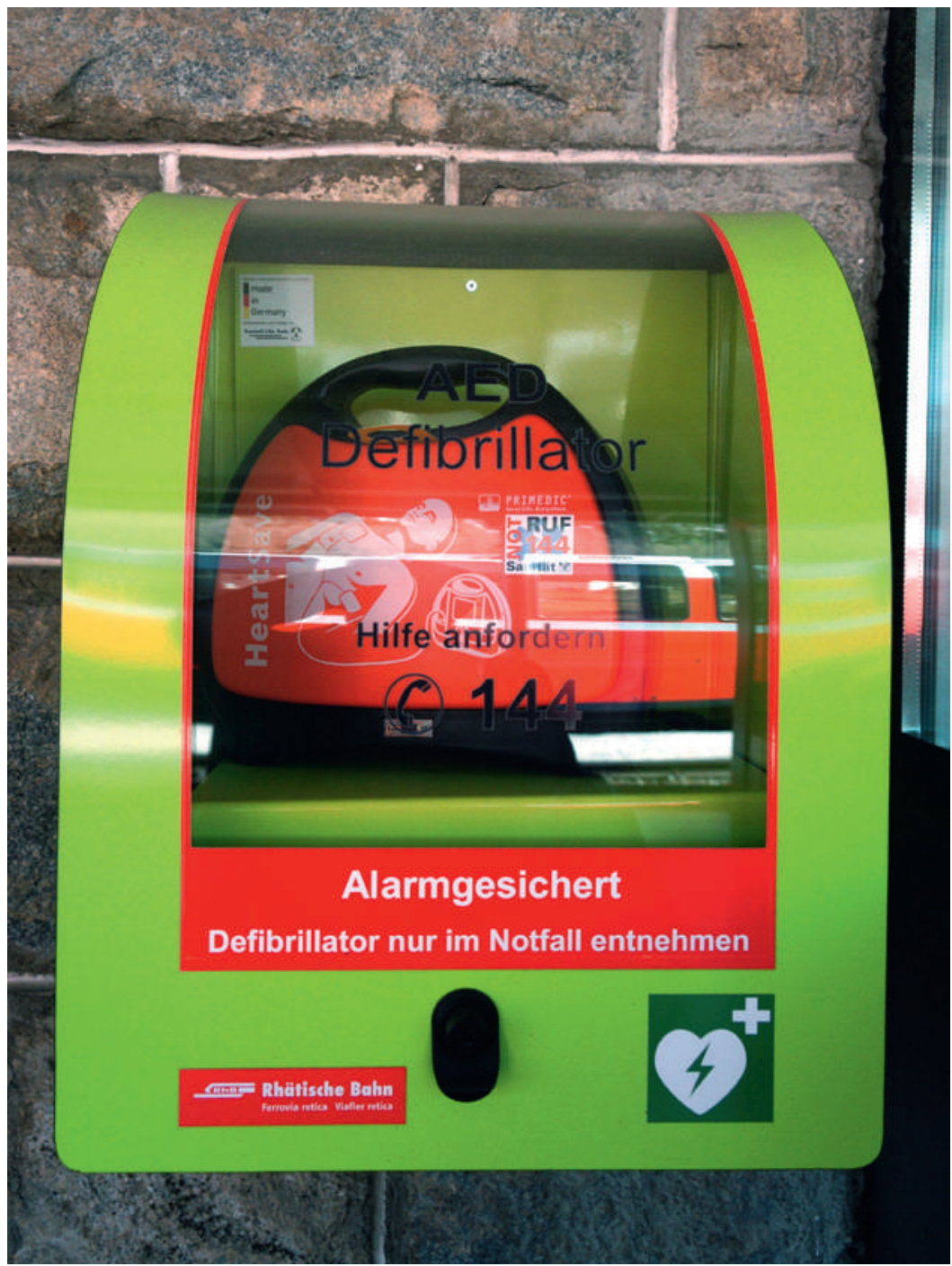

Mit dem Automatisierten Externen Defibrillator am Bahnhof von St. Moritz können Menschen Leben retten.

\section{Kanton Tessin macht es vor: Reanimation bereits in der Grundschule}

Als Grund für die Vernachlässigung dieses wichtigen Themas durch die Politik werden oftmals die Kosten aufgeführt - eine kurzsichtige Denkweise, weil nicht gerettete Menschenleben einen erheblichen (verlorenen) Wert darstellen. Einzelinitiativen ist es zu verdanken, dass zum einen vorbildlich funktionierende Ersthelfer-Rettungen im Sinne des Erkennens der Situation, der Alarmierung und des sofortigen Beginns von BLS-Massnahmen durch unmittelbar Beteiligte entstanden sind, zum anderen aber auch telefonische BLS-Instruktionen im Notfall, öffentlich zugängliche Automatisierte Externe Defibrillatoren (AED) oder First-Responder-Systeme. Gelegentlich hat auch der unerwartete Kreislaufstillstand einer prominenten Person das öffentliche Interesse geweckt.
Nur wenn ein plötzlicher Kreislaufstillstand sofort erkannt und unverzüglich mit wirksamen BLS-Massnahmen begonnen wird, bestehen für den Patienten realistische Überlebenschancen. Die Bevölkerung muss deswegen flächendeckend und effizient in BLS geschult werden; dies umso mehr, als ein ausreichender BLS heute dank vereinfachter Richtlinien problemlos durchführbar ist. Dies kann bereits an den Schulen erfolgen, wie dies bereits von den Kantonen Obwalden und Tessin beispielhaft praktiziert wird. Im Tessin werden seit sechs Jahren alle Schüler im Abschlussjahr der Grundschule in BLS trainiert. Bei jährlich 3500 Grundschulabsolventen haben damit schon 21000 junge Menschen bereits frühzeitig die wichtigen Kenntnisse im Erkennen von Notfällen und in der richtigen Reaktion sowie die erforderlichen Fähigkeiten erworben. Sie sind so auch in der Lage, dieses Wissen in die Familie zu tragen. Könnte dies nicht ein Ansporn sein, in allen Kantonen eine BLS-Ausbildung in den Lehrplan aufzunehmen? Dies ist notabene eine Kampagne, die auch in Regionen der USA mit einer vorbildlichen Reanimations-Outcome-Bilanz wie Seattle im Bundesstaat Washington bereits vor mehr als 20 Jahren zielführend war.

\section{Defibrillatoren geplant aufstellen - und Umgebung einbeziehen}

Ein Automatisierter Externer Defibrillator (AED) sollte an prädestinierten Orten mit entsprechendem Publikumsverkehr wie Bahnhöfen, Schulen, Mehrzweckhallen oder Gemeindehäusern öffentlich und rund um die Uhr zugänglich sein. Alle öffentlichen und private AED sollten erfasst und sowohl von der SNZ als auch von privaten Nutzern (z.B. via Smartphone) abrufbar sein. Sinnvollerweise soll an diesen mit AED ausgerüsteten öffentlichen Standorten geeignetes Personal in BLS-/ AED-Massnahmen geschult werden: Security-Angestellte, Zugpersonal, Lehrkräfte, Hauswarte, Gemeindemitarbeitende usw. In einer Notfallsituation kann eine Privatperson oder die SNZ somit schnell den nächstgelegenen AED ermitteln; parallel kann die SNZ in BLSMassnahmen geschulte Personen mit dem AED an den Ereignisort zur Unterstützung derjenigen schicken, die bereits mit den BLS-/AED-Massnahmen begonnen haben.

Das alleinige planlose Aufstellen von AED an Orten ohne hinterlegtes Einsatzkonzept ist nicht sinnvoll. Nur wenn gleichzeitig unverzügliche Hilfe mit BLSMassnahmen geleistet wird, ist das Instrument AED in der Folge wirksam. Dies sollten die derzeitigen und zukünftigen Besitzer von AED (Gemeinden, Firmen, Vereine) berücksichtigen; sie müssen von der Bedeutung 
des Gesamtsystems BLS-AED überzeugt sein. Informationen zu BLS-/AED-Aktionen inkl. des zugrunde liegenden Algorithmus stehen auf der Website des SRC (www.resuscitation.ch - Richtlinien 2010) zur Verfügung.

\section{Ein Automatisierter Externer Defibrillator (AED) sollte an Orten mit Publikumsverkehr rund um die Uhr zugänglich sein.}

An Orten, die ausserhalb der IVR-Hilfsfristen liegen, sollten First-Responder-Gruppen gebildet und durch die Behörden unterstützt werden. Interessant ist auch die von einigen Kantonen (z.B. Tessin) und Städten (z.B. Zürich) durchgeführte Schulung des gesamten Polizeikorps in BLS-Massnahmen und Ausrüstung der Patrouillenfahrzeuge mit AED und teilweise Beatmungshilfen

\section{Unterstützung durch Ärzteschaft und Politik notwendig}

Einzelinitiativen haben gezeigt, dass mit der Ersthelfer-Rettung Menschen gerettet werden können. Jetzt ist auch die Unterstützung der Ärzteschaft gefragt, damit schweizweit die Überlebensraten nach Kreislaufstillstand nachhaltig verbessert werden können. Unabdingbar ist zusätzlich zum politischen Support aber auch die berufsspezifische Diskussion in den kantonalen Ärztegesellschaften und unter den Kantonsärzten. Die eindrücklichen Outcomezahlen aus dem Kanton Tessin sind darüber hinaus nur dank eines kantonalen Reanimationsregisters bekannt, das im Einklang mit den gesetzlichen Bestimmungen und nach Prüfung der kantonalen Ethikkommission erstellt wurde. Will man die Effektivität der Rettungskette belegen, muss man auf ein solches Register zurückgreifen können. Dies trifft gleichermassen für die Rettungskette der ganzen Schweiz zu; ein gesamtschweizerisches Reanimationsregister wie beispielsweise SwissReCA nach dem Tessiner Vorbild sollte deshalb durch die Ärzte- schaft und die politisch Verantwortlichen gezielt unterstützt werden.

Kernsätze:

- Mit BLS/AED durch Ersthelfer kann die Überlebenschance nach einem Kreislaufstillstand deutlich erhöht werden.

- BLS-/AED-Unterricht soll in den Lehrplan der Schulen aufgenommen werden.

- Das alleinige Aufstellen von AED ist nicht ausreichend: Sie müssen in ein sinnvolles Nutzungskonzept integriert werden - und geeignete Zielgruppen im Umfeld von aufgestellten AED in BLS geschult werden.

- Die systematische Erfassung und Deklaration aller öffentlichen, halböffentlichen und privaten AED ist die Voraussetzung für deren gezielten Einsatz durch SNZ und Privatpersonen.

- First-Responder-Systeme sollen in geeigneten Regionen aufgebaut und unterstützt werden.

- Der Aufbau eines gesamtschweizerischen Reanimationsregisters ist sinnvoll und muss von der Ärzteschaft und der Politik begleitet werden.

\section{Referenzen}

1 Die Gleichstellung von Frau und Mann ist für die Autoren eine Selbstverständlichkeit. Der besseren Lesbarkeit wegen wird im Text nur die männliche Form verwendet.

2 Der Utstein-Style ist eine Anweisung für das einheitliche Reporting des Herz-Kreislauf-Stillstandes. 1990 fand in Norwegen in der Abtei von Utstein ein internationales Treffen der an Reanimationsforschung beteiligten Organisationen statt. Die Mitglieder einer Arbeitsgruppe der American Heart Association, des European Resuscitation Councils, der Heart and Stroke Foundation of Canada sowie des Australian Resuscitation Councils haben hierbei Empfehlungen zur einheitlichen Datenerfassung bei Herzstillständen erarbeitet.

3 Drolshagen S. Mit System gegen den Herztod. Rettungsdienst 2014;37:722-5

4 First Responder: Diese sind oft als Gruppen organisiert, welche parallel zum Rettungsdienst aufgeboten werden. Solche Gruppen sind in der Schweiz meist im Milizsystem (ähnlich einer Ortsfeuerwehr) organisiert. Dadurch kann ein Patient bereits vor dem Eintreffen des Rettungsdienstes erstversorgt werden. Die First Responder leisten erweiterte Erste Hilfe entsprechend ihrer Ausbildung und Ausrüstung. In der Schweiz haben sich First-Responder-Systeme in weniger dicht besiedelten Gebieten gut bewährt, gewinnen aber auch in Agglomerationen zunehmend an Bedeutung. 